\title{
An Adaptive Fair-Distributed Scheduling Algorithm to Guarantee QoS for Both VBR and CBR Video Traffics on IEEE 802.11e WLANs
}

\author{
Saeid Montazeri, ${ }^{1}$ Mahmood Fathy, ${ }^{2}$ and Reza Berangi ${ }^{2}$ \\ ${ }^{1}$ Computer Group, Islamic Azad University, KhomeiniShahr Branch, Khomeinishar 84175/119, Iran \\ ${ }^{2}$ Department of Computer Engineering, Iran University of Science and Technology, Tehran 16846-13114, Iran \\ Correspondence should be addressed to Saeid Montazeri, s.montazeri@iaukhsh.ac.ir
}

Received 2 October 2007; Revised 15 February 2008; Accepted 16 April 2008

Recommended by Jianfei Cai

Most of the centralized QoS mechanisms for WLAN MAC layer are only able to guarantee QoS parameters for CBR video traffic effectively. On the other hand, the existing distributed QoS mechanisms are only able to differentiate between various traffic streams without being able to guarantee QoS. This paper addresses these deficiencies by proposing a new distributed QoS scheme that guarantees QoS parameters such as delay and throughput for both CBR and VBR video traffics. The proposed scheme is also fair for all streams and it can adapt to the various conditions of the network. To achieve this, three fields are added to the RTS/CTS frames whose combination with the previously existing duration field of RTS/CTS frames guarantees the periodic fair adaptive access of a station to the channel. The performance of the proposed method has been evaluated with NS-2. The results showed that it outperforms IEEE 802.11e HCCA.

Copyright (C) 2008 Saeid Montazeri et al. This is an open access article distributed under the Creative Commons Attribution License, which permits unrestricted use, distribution, and reproduction in any medium, provided the original work is properly cited.

\section{INTRODUCTION}

The wireless LAN (WLAN) systems have received increasing popularity in recent years because they are cost effective, comfortable, and have high capacity. On the other hand, using video applications has been very popular in recent years. Therefore, effective using of video streams over the WLANs is an obligation these days. To achieve this goal, QoS parameters should be supported over WLANs.

Supporting QoS requirements in WLANs can be done in two ways: prioritized QoS and guaranteed QoS. A prioritized-QoS WLAN can only prioritize between different traffic streams while a guaranteed-QoS WlAN can guarantee QoS parameters such as delay, jitter, and throughput for traffic streams. Implementing QoS, either prioritized or guaranteed, is a challenge in WLAN because there are a large number of streams with different QoS requirements in a WLAN. Also some QoS requirements have variable characteristics during the time like a VBR video traffic. These characteristics lead to an adaptive QoS supporting approach in WLANs. In addition to the large number of streams and QoS requirements which vary during the time, wireless channel capacity is limited and must be shared among streams fairly. Thus, an adaptive fair algorithm which can guarantee QoS parameters is necessary in WLANs.

IEEE task group "e" worked on the support of QoS in a new standard, called IEEE 802.11e [1]. It introduces a new access method called hybrid coordination function (HCF), which combines functions from the DCF and PCF mechanisms in IEEE 802.11. HCF has two access mechanisms: enhanced distributed channel access (EDCA) and controlled channel access mechanism (HCCA). These two methods support QoS, which will be described further. In the HCCA, there is a scheduler for scheduling different traffic streams (TSs) on different stations. The HCCA can guarantee QoS parameters but it needs a centralized device that is called point coordinator (PC). On the other hand, the EDCA which does not use any PC could not guarantee QoS parameters. It can only operate for high-priority traffics sufficiently so it is not a fair method. In addition, both HCCA and EDCA have to tolerate high overhead to adapt to the network conditions.

Many works have been done to improve QoS in the IEEE 802.11e MAC layer. These works can be divided into two 
categories: the works that improve QoS distributively and the works which improve QoS by using PC.

In [2], the authors proposed a new adaptive fairdistributed method. This method enhances the EDCA of IEEE $802.11 \mathrm{e}$ by increasing the contention window when the channel is busy. It also uses an adaptive fast backoff mechanism when the channel is idle. They computed an adaptive backoff threshold for each priority level by taking into account the channel load. In [3], the authors proposed a fully distributed MAC adaptation method. They achieve this by updating the MAC layer parameters like contention window based on the network condition. Adaptive EDCA is a new method based on the IEEE 802.11e EDCA that is proposed in [4]. The main idea in this method is to decrease CW $[i]$ after a successful transmission and increase it after a collision slower than it is done in the EDCA. Also it takes into account both the network condition and application requirements. An improved EDCA is achieved in [5] by using the new backoff algorithm called agedependent backoff (ADB). ADB changes the persistence factor by using the age of packets in the transmission queue and their lifetime. In [6], the authors proposed a mechanism called A-DRAFT that supports both absolute and relative throughputs in adaptive distributed manner. This mechanism also provides fair throughput support with low variation. In [7], a new mechanism called differentiated service EDCA (DSEDCA) was proposed to provide both strict priority and proportional fair service for IEEE 802.11 WLANs. In this mechanism, resource is allocated to flows of higher priority, then the remaining bandwidth is shared proportionally among the other service class according to their assigned weights. In [8], authors proposed a surplus TXOP diverter (STXD) scheduling algorithm which allows each flow to exploit its granted TXOP time to reduce the delay when burst packets arrival.

In [9], authors proposed a new scheduling algorithm in link layer to support multimedia services with guaranteed QoS in WLAN. Their scheduling algorithm is based on the HCF. It reduces average packet loss ratio by setting constant bit-rate (CBR) RT to the highest priority followed by VBR RT, and after all NRT level. It also uses idle time, while satisfying required rate allocation, transmission delay bound, and system throughput. In [10], a fair QoS agent (FQA) is proposed to provide per-class QoS enhancement and per-station fair channel access simultaneously. Authors put the FQA algorithm above the MAC layer which enables algorithm to be implemented without any change in the MAC layer. Their algorithm satisfies the fairness in WLAN MAC layer. In [11], a novel QoS capable station (QSTA) uplink scheduler along with a QoS capable AP (QAP) HCF scheduler can provide the QoS requirement of delay bound for multimedia applications. In [12], the authors proposed a new scheduling algorithm for IEEE 802.11e which they called FHCF. It outperforms IEEE 802.11e HCF especially for VBR traffic. It uses queue length estimation to tune time allocation to stations. A new scheduling algorithm has been proposed in [13] which enables the IEEE 802.11e scheduler to work with different SIs for different TSs in the stations. In [14], the authors proposed a dynamic bandwidth allocation algorithm along with the measurement-based call admission control algorithm which can provide delay guarantee for real-time flow. It uses a classic feedback control system. There is an enhancement for IEEE 802.11e HCF in [15] that improves the admission control unit of HCF. By this method, each priority has a certain and limited amount of resources.

Although all distributed methods in [2-8] can improve EDCA in IEEE 802.11e, they can not guarantee QoS parameters in WLANs. However, methods in [9-15] can guarantee QoS in WLANs, they need point coordinator and cause high overhead to guarantee QoS parameters for VBR video traffic. This paper proposes an adaptive fair-distributed scheduling algorithm (AFDSA) [16] for both VBR and CBR video traffic streams in WLANs. AFDSA is a distributed method that operates better than centralized methods in all fields especially for VBR video traffics with low overhead.

The rest of the paper is organized as follows. The IEEE $802.11 \mathrm{e}$ is introduced in Section 2. The properties of AFDSA algorithm are described in Section 3. Section 4 describes the simulation results. The conclusion follows in Section 5.

\section{IEEE 802.11e MAC}

Hybrid coordination function (HCF) of IEEE 802.11e MAC has both contention-based access method and polling-based access method. EDCA introduces the concept of access categories (ACs), which can be considered as instances of the DCF access mechanism. It provides support for the prioritized delivery at each station.

\subsection{Enhanced distributed channel access (EDCA)}

Like DCF, EDCA uses CSMA/CA protocol to access the wireless media. It only operates during CP. In EDCA method, each AC within the stations contends for transmission opportunity (TXOP) independently. TXOP is defined as the interval of time when a particular station has the right to initiate the transmission onto the wireless channel. Each AC starts the backoff after detecting the channel to be idle for a time interval equal to the arbitration interframe space (AIFS). Each AC has its AIFS which depends on the assigned priority. Figure 1 demonstrates the eight different queues for eight ACs.

Each $\mathrm{AC}$ has its own queue, $\mathrm{CW}_{\min }[\mathrm{AC}], \mathrm{CW}_{\max }[\mathrm{AC}]$, and $\mathrm{PF}[\mathrm{AC}]$. Figure 2 shows the different ways to provide service differentiation.

For each AC, backoff is generated in the range of [1, $\mathrm{CW}[\mathrm{AC}]+1]$. The initial value for the $\mathrm{CW}$ is $\mathrm{CW}_{\min }[\mathrm{AC}]$. $\mathrm{CW}$ is increased whenever the node involves in a collision by (1) up to $\mathrm{CW}_{\max }[\mathrm{AC}]$ :

$$
\text { newCW }[\mathrm{AC}]=((\text { oldCW }[\mathrm{AC}]+1) * \mathrm{PF}[\mathrm{AC}])-1 \text {, }
$$

where PF is the persistence factor, which equals 2 by default. It determines the degree of increase for the $\mathrm{CW}$ when 


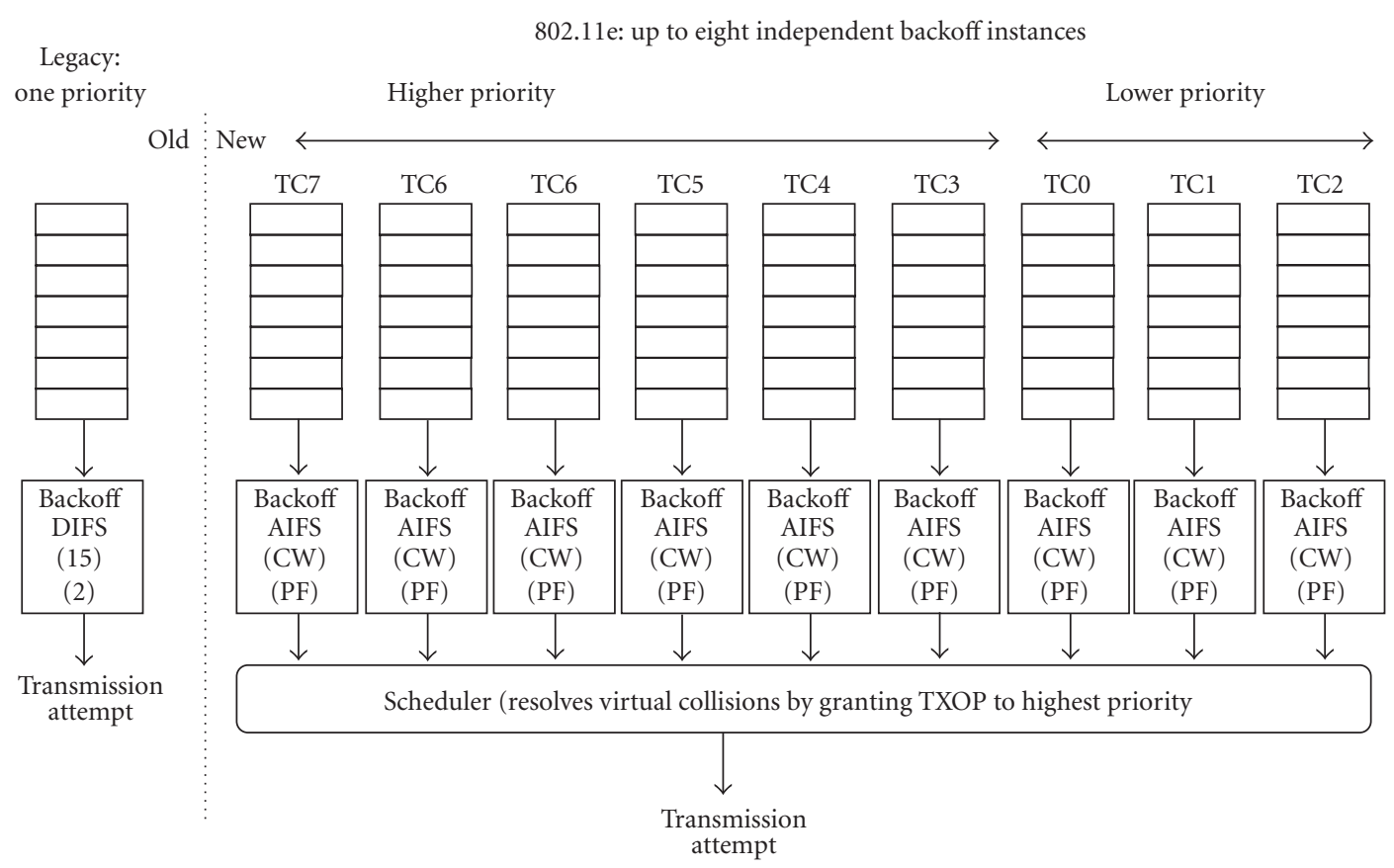

Figure 1: Old DCF and EDCA.

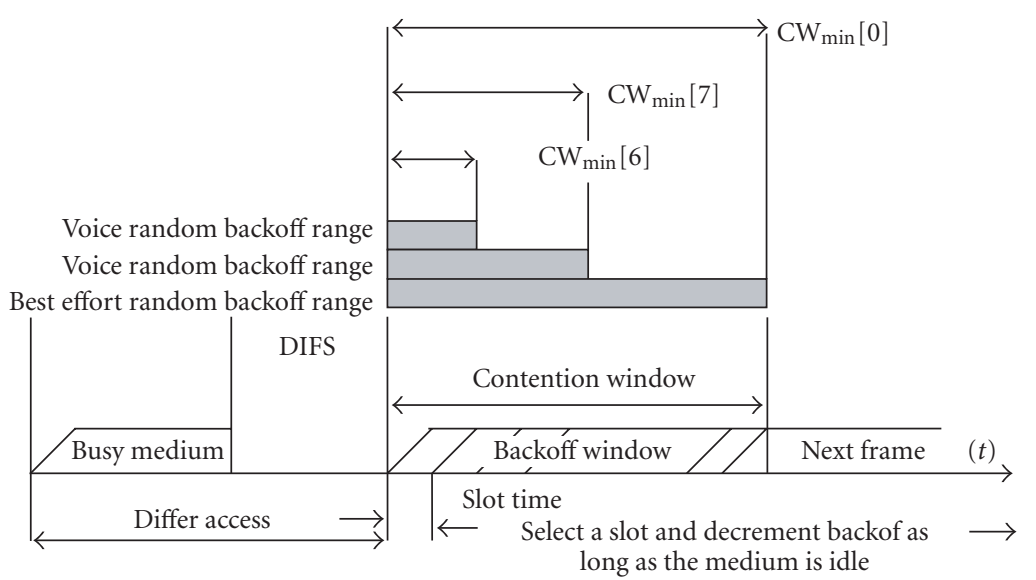

FIgure 2: Different AIFS for different priorities.

a collision happens. EDCA can only differentiate between different priorities.

\subsection{HCF controlled channel access (HCCA)}

In IEEE 802.11e standard, the polling-based scheme of 802.11 is extended in the form of HCCA, in which there is a hybrid coordinator (HC) usually colocated with a QoS AP (QAP). HC can access channel after waiting for a time which is shorter than each AIFS and DIFS. Thus, HC can get the channel in both CFP and CP. During CP, TXOP for each station can be received in two ways: by using EDCA rules or by receiving a poll from HC (polled_TXOP). During CFP, TXOP is determined only by HC with poll frame. CFP is ended by a CF_end frame which is transmitted by HC.

\subsection{11e HCF scheduling scheme}

The HCF has a simple scheduler in IEEE 802.11e. If a QoSenhanced station (QSTA) needs a strict QoS support, it should send a QoS requirement packet to the QAP while the QAP can allocate the corresponding channel time for different QSTAs according to their requirements. Figure 3 shows the new beacon interval of 802.11e, CFP, and CP. The QAP can operate in both CFP and CP. During the CP, the QAP can start several contention-free bursts at any time to control the channel which are called controlled access periods (CAPs).

If a station requires a contention-free access to the channel by getting TXOP, it should send a QoS request frame to the QAP containing several parameters. These parameters are mean data rate of the application, the maximum service 


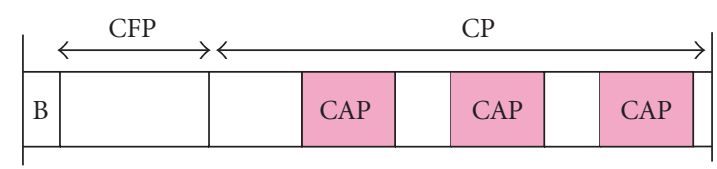

Figure 3: CFP, CP, and CAPs in the 802.11e.

interval (MSI) and MAC service data unit (MSDU) size. Then the QAP calculates the TXOP in two steps. In the first step, it determines the minimum value of all MSIs required by different traffic streams. Then, it chooses the highest submultiples' value of the $802.11 \mathrm{e}$ beacon interval duration (duration between two beacons) as the selected SI which is less than the minimum of all requested MSIs. This selected SI is the time between two successive TXOPs for all streams. Since it is less than or equal to all MSIs, it is guaranteed that every station with different streams can reach desired MSI for their streams. In the second step, the QAP calculates the TXOP for each TSs in different QSTAs. Calculated TXOP should correspond to the duration required for transmitting all packets that is generated during one SI by the specific TS. Figure 4 shows the CPs, CFPs, selected SI, and EDCA time.

Equations (2) and (3) determine the TXOP, where $\rho$ is the mean data rate of the application, and $L$ is the MAC service data unit (MSDU) size:

$$
N_{i}=\left\lceil\frac{\mathrm{SI} \times \rho_{i}}{L_{i}}\right\rceil .
$$

Here, $N_{i}$ is the number of packets that is generated during an SI for the $i$ th priority. $R$ is the physical transmission rate, $M$ is the size of maximum MSDU (2304 bytes), and $O$ determines the overhead in time units:

$$
\operatorname{TXOP}_{i}=\max \left(\frac{N_{i} \times L_{i}}{R_{i}}+O, \frac{M}{R_{i}}+O\right) .
$$

It can be easily deduced that the $\mathrm{TXOP}_{i}$ is the time required to send $N_{i}$ packets for a specific application.

\section{THE PROPOSED ADAPTIVE FAIR-DISTRIBUTED SCHEDULING ALGORITHM (AFDSA)}

\subsection{Concept}

All centralized channel access methods in WLANs, which are able to guarantee QoS parameters, have one PC that knows the QoS requirements of all TSs. These requirements, which are sent by each station to the PC before starting a transmission, enable the PC to schedule all TSs. The PC can manage QSTAs and guarantee QoS because of its awareness about the requirements of all traffic streams and its ability to get the channel in desirable time. As a matter of fact, PC polls the stations in a proper way by using its knowledge about the network condition. On the other hand, the distributed methods do not have such a PC or equivalent device to gather information and guarantee QoS by managing QSTAs.

The most important characteristic of our approach is to distribute the necessary information (which is different from the one in IEEE 802.11e) among QSTAs to make aware all stations about the network situation. The proposed AFDSA employs the RTS/CTS feature in IEEE 802.11 with some changes. By using this feature, we can reduce the overhead which is required for distributing QoS parameters. The RTS/CTS handshaking mechanism is used to solve the hidden terminal (hidden node) problem in IEEE 802.11 WLANs. A hidden node problem happens when two stations that communicate with a common station are not able to hear each other so their packets collide. Figure 5 demonstrates RTS/CTS packets in which the frame control field is related to the control functions, Duration field contains a value that shows the duration of a transmission, RA is the receiver address, TA is the transmitter address, and FCS is the frame check sequence of the packets.

A RTS/CTS protocol initiates with sending an RTS frame to the receiver (Figure 6). A transmission only starts when a CTS frame is replied to by the receiver. All the stations, which receive one of these frames, understand that a transmission will start and continue for duration equal to the duration field in the RTS/CTS frames. They set their network allocation vector (NAV) to the proper value to prevent themselves from disturbing transmission.

This local hand shaking between transmitter and the receiver provides an excellent opportunity to distribute necessary information to guarantee QoS parameters. To achieve this, the proposed AFDSA uses a modified RTS/CTS protocol with additional fields in the original IEEE 802.11 RTS/CTS frames. The new fields, that is, CurrentSI, FutureSI, and remainderSI, as shown in Figure 7, are added to both RTS/CTS.

What are the CurrentSI, FutureSI, and remainderSI? Before defining these fields, we should define service interval. Service interval is the time between two successive TXOPs that belong to the specific traffic stream. AFDSA uses this concept for the service interval (SI). When a WLAN works with a specific SI, a station can reach the channel for TXOP seconds and it is repeated each SI seconds. In AFDSA, CurrentSI is the service interval that the WLAN is working with at the time of transmission; FutureSI is the service interval that WLAN will work with after ending present SI; and remainderSI indicates the time that is remaining until the end of this service interval (after receiving the RTS/CTS packets). Also TA (transmitter address) is added to the CTS frames for the future development. The protocol needs two timers; a duration timer and a service interval (SI) timer.

The protocol sends the RTS/CTS frames only before the first few packets in each transmission to reduce the transmission overhead. The exact number of required RTS/CTS packets will be calculated in the next section. The QoS parameters can be only guaranteed for a traffic stream (TS) when the TS can have access to the channel for a special duration with a specific SI. Duration for $i$ th TS in the $j$ th QSTA can be obtained from

$$
\begin{aligned}
D_{i}^{j}= & N_{i}^{j} *\left(\frac{M_{i}}{R}+2 \text { SIFS }+ \text { ACK }_{\text {time }}\right) \\
& +{\text { RTSNumber } *\left(\text { RTS }_{\text {time }}+\text { CTS }_{\text {time }}+2 \text { SIFS }\right)} \\
& -\left(\text { RTS }_{\text {time }}\right)
\end{aligned}
$$



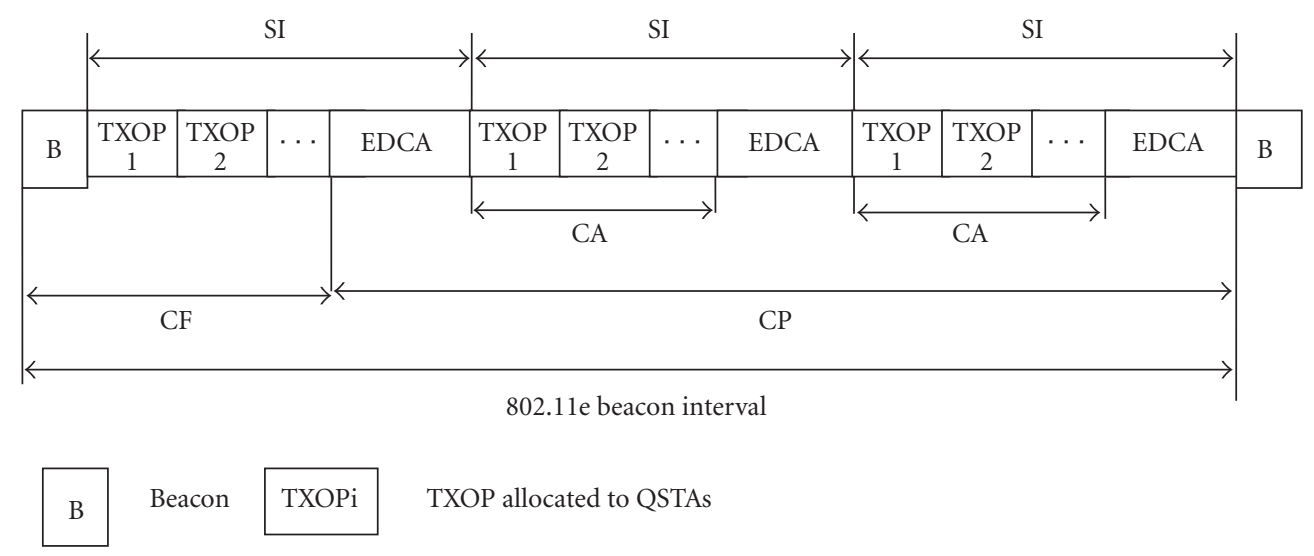

Figure 4: Structure of the 802.11e beacon interval.

\begin{tabular}{|l|l|l|l|l|}
\hline $\begin{array}{c}\text { Frame } \\
\text { control }\end{array}$ & Duration & RA & TA & FCS \\
\hline
\end{tabular}

RTS frame

\begin{tabular}{|c|c|c|c|}
\hline $\begin{array}{c}\text { Frame } \\
\text { control }\end{array}$ & Duration & RA & FCS \\
\hline
\end{tabular}

CTS frame

FIgURE 5: RTS/CTS frame structure.

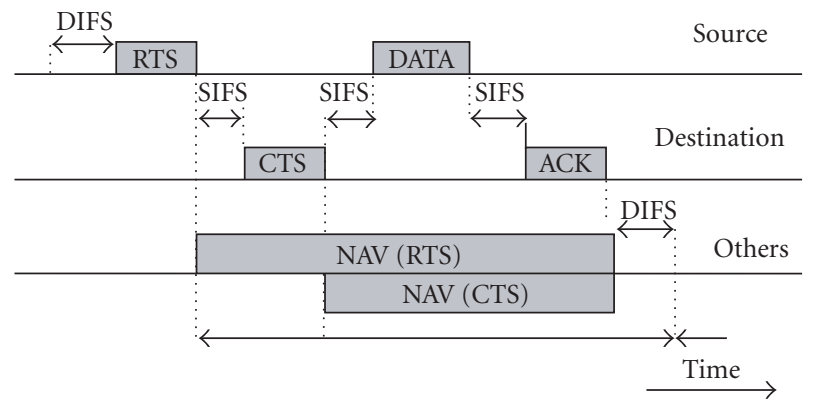

FIgURE 6: RTS, CTS, data, and ACK frames sequence.

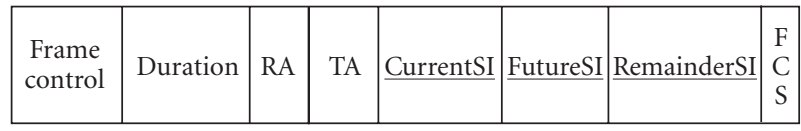

FIgURE 7: New RTS/CTS frame structure.

where, $D_{i}^{j}$ is the time required to transmit $N_{i}^{j}$ packets with the length $M_{i}$, the physical rate $R$, plus the time required to transmit RTSNumber of RTS and CTS frames. Here, $N_{i}^{j}$ is the number of packets in the queue of $i$ th TS in the $j$ th QSTA at the time of calculating $D_{i}^{j}$.

The question that is to be answered here is how to guarantee $D_{i}^{j}$ repeats every SI seconds for the $i$ th TS in the jth QSTA, without disturbing other QSTAs with different TSs requirements. Suppose that ith TS in the jth QSTA is the first one that starts the transmission in the WLAN with using EDCA method. It calculates $D_{i}^{j}$ and puts it in the duration field of RTS. Then it sets the CurrentSI and FutureSI with maximum service interval for $i$ th TS. The $j$ th QSTA transmits
RTS frame and waits for receiving CTS. Destination receives the RTS and calculates the duration field for CTS by using

$$
\text { Duration }_{\text {CTS }}=\text { Duration }_{\mathrm{RTS}}-\left(\mathrm{SIFS}+\mathrm{CTS}_{\text {time }}\right) \text {. }
$$

Then, it transmits the CTS frame to the $j$ th QSTA. All the QSTAs that receive the RTS or CTS understand a new transmission will be started with the specific length (duration field in the RTS/CTS frames) and will be repeated with a specific period (CurrentSI field in the RTS/CTS frames). Therefore, they reserve this time for station $j$ by setting and starting their duration timers with the duration field of RTS/CTS. They also set and start SI timers with the CurrentSI field of RTS/CTS as well as saving FutureSI field of RTS/CTS for the next SI timer restart. Finally, when source receives the CTS frame, it transmits data frame.

The duration field of RTS is updated by the value of the duration timer in the source station. After this, all stations have reserved the allocated turn for the $j$ th station and they keep silent during this time. It is done by using an array. Each station has an array and saves the sequence of turns in it. If a station saves 0 in the $i$ th place in array, it means that, in the SI, the ith turn is reserved for another station, yet if it saves 1 in the $i$ th place in array, it means that in the SI the $i$ th turn is reserved for itself. The exact duration is announced in the duration field of RTS/CTS by the $j$ th station and other stations do not need to save the value of duration field. Therefore, duration field can be varied and updated each time. It is perfect for VBR video traffics and can adapt itself to the network condition.

After finishing $D_{i}^{j}$, all QSTAs start to compete for accessing the channel based on EDCA method. Suppose that $k$ th QSTA gets the channel for its $l$ th TS. It fills the duration field by using (5), and sets the CurrentSI with the value of CurrentSI of jth QSTA. However, it sets the FutureSI field with maximum service interval that $l$ th TS required when 
it is equal or less than previous FutureSI (related to $i$ th TS in the $j$ th QSTA). So, all the QSTAs that receive the new RTS/CTS understand that they must initialize their SI timer with FutureSI field of new RTS/CTS at the end of current SI. Therefore after a number of SIs, the network works with the sufficient SI. This SI is the minimum of maximum service intervals for all TSs.

After finishing the first SI, all stations check whether they have the first turn. They do this by using their arrays. The station which finds that it has the first turn, that is, $j$ th station, starts to calculate the $D_{i}^{j}$ and transmit the RTS. All the other stations keep quiet and wait until they receive an RTS or CTS frame. If a station receives an RTS/CTS, it starts its duration timer. When a duration timer goes zero, it is the time to go to the next turn and search the array. Also requesting stations must only compete in the free time at the tail of current SI (as shown in Figure 8).

Now we can describe why AFDSA is sufficient for transmitting video traffics. Other distributed method, EDCA, can only prioritize between various kinds of streams. As depicted in Table 4, CBR video traffic has the lowest priority and after that comes the VBR video traffic. In a WLAN with different kinds of streams and using EDCA, the video streams can not adequately access the channel in competition with other types of traffics. On the other hand, HCCA method needs specific characteristics of a stream-like data rate and packet size to allocate channel to it. However, data rate and packet size vary during the time for VBR video traffics. As a result, PC in HCCA method can not allocate the accurate time to the VBR traffics since it is obliged to select one of the following two choices. The first one is to allocate the channel to the VBR streams based on the mean data rate. It leads to some dropping packets, wasted channel, and increased jitter and delay because of the great changes in the amount of data. The other choice is to allocate the channel based on the peak data rate to prevent the packet loss. It causes to waste the channel much more than what it may happen in the first method.

AFDSA can adapt the allocated time of each station for transmitting packets by using number of packets that are available in the queue at the beginning of the $D_{i}^{j}$. This leads to improve the channel efficiently by letting the others to use the channel. This prevents packet dropping and channel wasting simultaneously.

The mentioned channel access process in AFDSA eliminates the need for a point coordinator, though each wireless station can act as an AP when it is connected to the wired network. This enhances the survivability of WLANs in case of an AP failure.

\subsection{Special situations}

This section reviews the performance of proposed protocol in special situation that might happen during a period in which a WLAN works.

\subsubsection{Missing the RTS/CTS}

It is very important for all the stations to be synchronized so that their SI timers start and finish on time. If a station
TABLE 1: Data transmission sequence for a specific TS in AFDGP.

\begin{tabular}{cccc}
\hline RTS $_{1}$ & SIFS & CTS $_{1}$ & SIFS \\
Data $_{1}$ & SIFS & Ack $_{1}$ & SIFS \\
RTS $_{2}$ & SIFS & CTS $_{2}$ & SIFS \\
Data $_{2}$ & SIFS & Ack $_{2}$ & SIFS \\
RTS $_{3}$ & SIFS & CTS $_{3}$ & SIFS \\
Data $_{3}$ & SIFS & Ack $_{3}$ & SIFS \\
$\vdots$ & $\vdots$ & $\vdots$ & $\vdots$ \\
RTS $_{\text {RTSNumber }}$ & SIFS & CTS $_{\text {RTSNumber }}$ & SIFS \\
Data $_{\text {RTSNumber }}$ & SIFS & Ack $_{\text {RTSNumber }}$ & SIFS \\
Data $_{\text {RTSNumber+1 }}$ & SIFS & Ack $_{\text {RTSNumber+1 }}$ & SIFS \\
Data $_{\text {RTSNumber+2 }}$ & SIFS & Ack $_{\text {RTSNumber+2 }}$ & SIFS \\
Data $_{\text {RTSNumber+3 }}$ & SIFS & Ack $_{\text {RTSNumber+3 }}$ & SIFS \\
\hline
\end{tabular}

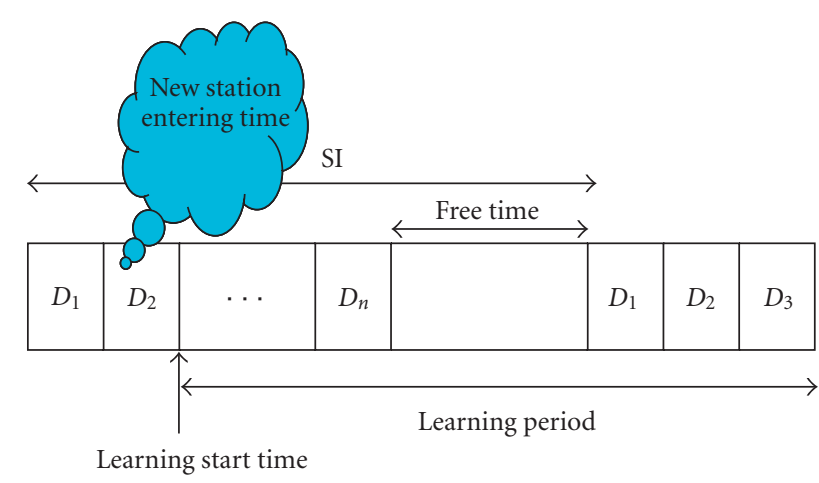

Figure 8: Free time and learning period.

misses the RTS or CTS, it must wait until it receives the next RTS or CTS. By receiving the next RTS or CTS, it can use the duration and remainderSI fields to synchronize itself with the others because these fields are always up to date. The process of sending and receiving RTS/CTS repeats RTSNumber times to assure that all the active stations in the communication range have received at least one RTS or CTS. After which only data frames will be transmitted. The RTSNumber depends on the BER of the channel and increases with increasing the BER. In our simulation, RTS number is set to 2. Table 1 shows data transmission sequences and shows the impact of RTSNumber on the data transmission.

\subsubsection{Entering a new station to the working WLAN}

A new station needs remainderSI, FutureSI, and CurrentSI to synchronize with a working WLAN. So it must wait until it receives at least one RTS or CTS and it must wait at least one SI to learn about network condition. This SI which is referred to as the learning period is shown in Figure 8. Any new entering station is prevented to send data during its learning period. Not having permission to send data in learning period is a rule in AFDSA. It can only access the channel based on the EDCA rule in the free time after the learning period and after it receives at least one RTS or CTS packet too. 


\subsubsection{Removing a duration between other durations}

If a station stops using its allocated turn related to a specific TS (e.g., ith TS), it must send special RTS to the receiver RTSNumber times. Receiver replies to this special RTS by a special CTS frame. These special RTS/CTS frames mean that duration will not continue any more. So other stations that receive these frames understand that they must remove this special duration and its turn. The RTS duration field is calculated by

$$
\begin{aligned}
& \text { Duration } \\
& =\text { RTSNumber } *\left(\text { RTS }_{\text {time }}+\text { CTS }_{\text {time }}+2 \text { SIFS }\right)\left(\text { RTS }_{\text {time }}\right) \text {. }
\end{aligned}
$$

This process will be repeated RTSNumber times to assure that all the listening stations in the WLAN receive at least one RTS or CTS frame. In as much as the RTSNumber depends on the BER, it is possible to set its value based on the probability of missing RTS or CTS by one station. It is possible for this value to be less than a special limit. A WLAN with $m$ station which has an active flow and RTSNumber gives this probability through (7) to (9)

$$
\begin{gathered}
P_{\mathrm{RTS} / \mathrm{CTS}}^{f}=1-(1-\mathrm{BER})^{\mathrm{RTS}_{\text {Length }}} \approx \mathrm{RTS}_{\text {length }} * \mathrm{BER}, \\
P_{\text {Station }}^{f}=\left(P_{\mathrm{RTS} / \mathrm{CTS}}^{f}\right)^{\mathrm{RTSNumber}}, \\
P^{f}=1-\left(1-P_{\text {Station }}^{f}\right)^{n-1} \approx(n-1) * P_{\text {Station }}^{f},
\end{gathered}
$$

where $P_{\mathrm{RTS} / \mathrm{CTS}}^{f}$ is the corruption probability of an RTS or CTS frame, $P_{\text {Station }}^{f}$ is the probability that a station does not receive any of the sent RTSs or CTSs, and $P_{f}$ is the probability of not receiving even one RTS/CTS by a station among $m-1$ listening stations. By using Table 4 for RTS $_{\text {length }}$ and assuming that BER is equal to $10^{-5}[1], P_{\mathrm{RTS} / \mathrm{CTS}}^{f}$ will be 0.00224 . The power factor in (8) is RTSNumber rather than $2 *$ RTSNumber because in severe situations the listening station may only receive either RTS or CTS because of being in the signal range of either RTS transmitter or CTS transmitter. For a WLAN with 200 active flows and RTSNumber $=3$, the $P_{f}$ is equal to $2.2 * 10^{-6}$.

\subsection{AFDSA scalability}

Since the AFDSA is a distributed algorithm, it has a good scalability. It can accept new stations until the channel saturates, or there is no bandwidth to assign. Since new stations only compete for TXOPs in free time, as depicted in Figure 8, no new station can reach channel if there is not any free time available. So if the number of stations is increased, network is accessible only for the number that can send their packets with adequate quality of service. It means by using AFDSA, a station can either send their packets with proper QoS or cannot have access to the channel for sending its packets. Perhaps it seems to be an unfair algorithm. However, the authors think assigning the network channel to a limited number of stations by good QoS parameters is better than sharing the channel among a large number of dissatisfied QoS stations.
TABLE 2: Scenario 1 nodes and traffic flows.

\begin{tabular}{ccccc}
\hline Node & Application & $\begin{array}{l}\text { Arrival } \\
\text { period } \\
(\mathrm{ms})\end{array}$ & $\begin{array}{l}\text { Packet } \\
\text { size } \\
\text { (bytes })\end{array}$ & $\begin{array}{l}\text { Sending } \\
\text { rate } \\
(\mathrm{kbps})\end{array}$ \\
\hline $1 \rightarrow 6$ & Audio & 4.7 & 160 & 64 \\
$7 \rightarrow 12$ & VBR video & $\approx 26$ & $\approx 660$ & $\approx 200$ \\
$13 \rightarrow 18$ & MPEG4 video & 2 & 800 & 3200 \\
\hline
\end{tabular}

TABLE 3: Traffic specification.

\begin{tabular}{lcccc}
\hline Traffic type & Priority & $\mathrm{CW}_{\text {Min }}$ & $\mathrm{CW}_{\text {Max }}$ & Max delay $(\mathrm{ms})$ \\
\hline Voice & 6 & 7 & 15 & 50 \\
VBR video & 5 & 15 & 31 & 100 \\
CBR video & 4 & 15 & 31 & 100 \\
\hline
\end{tabular}

TABle 4: The PHY and MAC layer parameters.

\begin{tabular}{lccc}
\hline SIFS & $16 \mu \mathrm{s}$ & CCA Time & $4 \mu \mathrm{s}$ \\
DIFS & $34 \mu \mathrm{s}$ & MAC header & 38 Bytes \\
ACK size & 14 bytes & PLCP header length & 4 bits \\
PHY rate & $36 \mathrm{Mp} / \mathrm{s}$ & Preamble length & 20 bits \\
Minimum bandwidth & $6 \mathrm{Mp} / \mathrm{s}$ & RTS length & 28 bytes \\
Slot time & $9 \mu \mathrm{s}$ & CTS length & 28 bytes \\
\hline
\end{tabular}

\subsection{AFDSA overhead}

AFDSA sends RTSNumber RTS/CTS in addition to the packets that must be sent. AFDSA overhead can be calculated by

$$
\begin{aligned}
O_{\text {Total }}=[ & \text { RTSNumber } * \frac{1 \mathrm{Sec}}{\mathrm{SI}} * \text { Numberof TotalFlows } \\
& \left.*\left(\mathrm{CTS}_{\text {Time }}+\mathrm{SIFS}+\mathrm{RTS}_{\text {Time }}+\mathrm{SIFS}\right)\right],
\end{aligned}
$$

where RTS $_{\text {Time }}$ is the time required to transmit RTS packet, $\mathrm{CTS}_{\text {Time }}$ is the time required to transmit CTS packet, RTSNumber is defined in Section 4.1, SIFS is the time between two successive transmissions as depicted in Table 1. Since AFDSA sends RTS/CTS packets only at the beginning of each transmission, one second is divided by SI to find the number of SI repeats in one second. Also the total number of flows, NumberofTotalFlows, is calculated by

$$
\text { Numberof TotalFlows }=\sum_{i=1}^{n} f_{i} \text {, }
$$

where $f_{i}$ is the number of flows in the $i$ th station. Since in our simulation $\mathrm{RTS}_{\text {Time }}=\mathrm{CTS}_{\text {Time }}=12 \mu \mathrm{s}$, SIFS $=16 \mu \mathrm{s}$, RTSNumber $=2$, and Numberof TotalFlows is 18 , the $O_{\text {Total }}$ found from (11) is equal to $40320 \mu \mathrm{s}$. This is the time that AFDSA consumes for transmission of RTS/CTS packets in one second (4 percent). As it is clear form (10) and (11), neither the number of stations nor the size of packets affects the overhead. Only the NumberofTotalFlows, selected SI and RTSNumber, can affect the overhead. It must be mentioned that the NumberofTotalFlows in a WLAN can be increased until the channel saturates. After that, increase in the number 
TABLE 5: Jitter for different types of traffic in different methods.

\begin{tabular}{lcccc}
\hline & HCCA & FHCF & AFDSA & EDCA \\
\hline Audio & $14.2(\mathrm{~ms})$ & $14.5(\mathrm{~ms})$ & $14.1(\mathrm{~ms})$ & $0.9(\mathrm{~ms})$ \\
VBR video & $460.57(\mathrm{~ms})$ & $14.7(\mathrm{~ms})$ & $19.4(\mathrm{~ms})$ & $3.2(\mathrm{~ms})$ \\
CBR video & $20(\mathrm{~ms})$ & $15.1(\mathrm{~ms})$ & $13.7(\mathrm{~ms})$ & $22.5(\mathrm{~ms})$ \\
\hline
\end{tabular}

of stations cannot influence the NumberofTotalFlows since the new stations can not access the channel. If these new stations are able to access the channel in a saturated manner, it is impossible to guarantee QoS parameters for any flow.

\section{SIMULATION RESULTS}

AFDSA is implemented using NS-2 simulator and compared with the three previously reported works for the distributed [2-8] and centralized [9-15] channel access mechanisms. Both distributed (EDCA) and centralized methods (HCCA) of $802.11 \mathrm{e}$ [1] are selected since they are widely used in the literature for comparison. The fair HCF (FHCF) proposed in [12] is also selected as the third scheme to compare with our method. Two kinds of simulation scenarios have been used. The first one contains 18 sources and one destination. The second contains 6 sources and one destination. In both scenarios, the destination is QAP that contains a PC to satisfy the requirements for HCCA and FHCF, yet it is an ordinary QSTA for our proposed method.

\subsection{Scenario 1}

In scenario 1, 6 QSTAs send a high-priority on/off audio traffic (64 kbps) each, another 6 QSTAs send a VBR video traffic (200 kbps of average sending rate) with medium priority each, and 6 QSTAs send a CBR MPEG4 video traffic (3.2 Mbps) with low priority each. Voice traffic is used to indicate that AFDSA in the presence of the high-priority traffic is still able to give desirable QoS parameters for both CBR and VBR traffics. Table 2 summarizes the different traffics used for this simulation. We model the audio flow by on/off source with parameters corresponding to a typical phone conversation [17]. UDP is used as transport protocol.

Figures 9 to 12 demonstrate the latency distribution of the simulated methods. It shows that AFDSA has a maximum latency for each traffic stream under its tolerable latency (Table 3 ). In contrast, VBR traffic latency in HCCA is uncontrollable and in EDCA exceeds the limit. Maximum VBR latency for the proposed method is 80 milliseconds but for FHCF is 50 milliseconds. This might seem to be an advantage for FHCF but it must be considered that FHCF is a centralized method that needs PC where AFDSA is a distributed algorithm that does not need any PC. Also the AFDSA latency is still lower than the tolerable latency for VBR video. These differences relate to the starting situations. FHCF starts with the SI $=50 \mathrm{~ms}$ and continues by this yet AFDSA starts with the SI $=100 \mathrm{~ms}$ and then changes it to $50 \mathrm{~ms}$. So, the grater SI belongs to the starting TS, that is, a VBR video stream which its maximum latency is 100 .

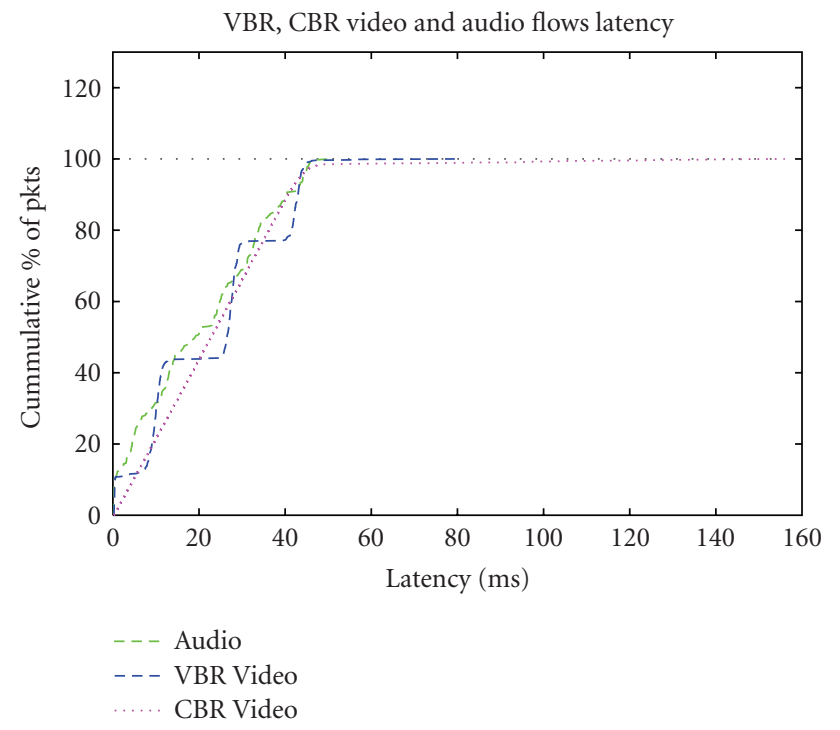

FIgURE 9: Latency distribution for FHCF.

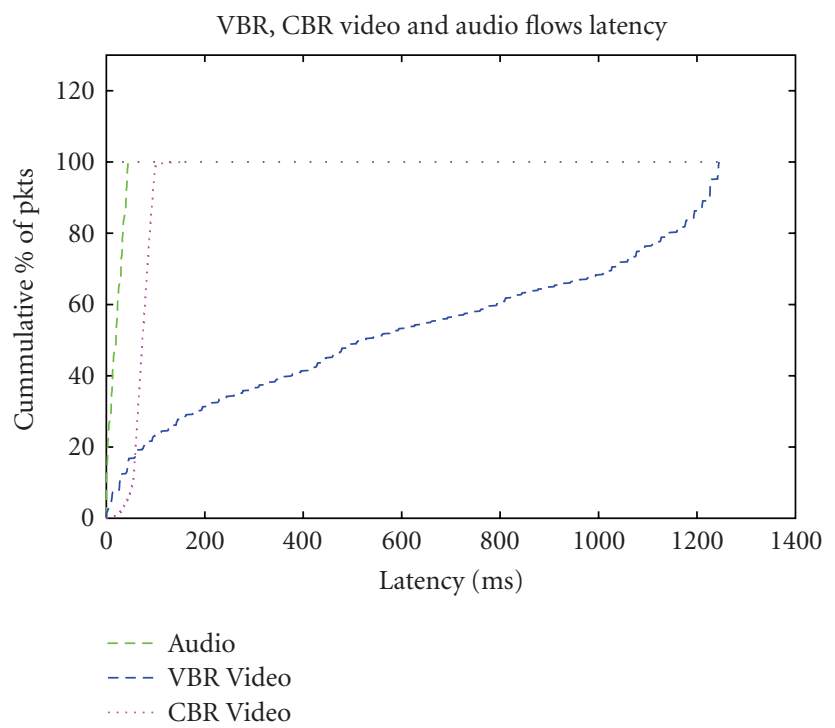

FIGURE 10: Latency distribution for standard HCF.

Therefore, AFDSA sets the currentSI to 100 then it changes it to 50 .

The same figure also shows that the latency distribution curve of the VBR flow has a stair shape. This shape relates to the packets interarrival time. Analysis of the VBR video trace file shows that the interarrival time of packets is 34 milliseconds (see Table 2) but some packets are received 


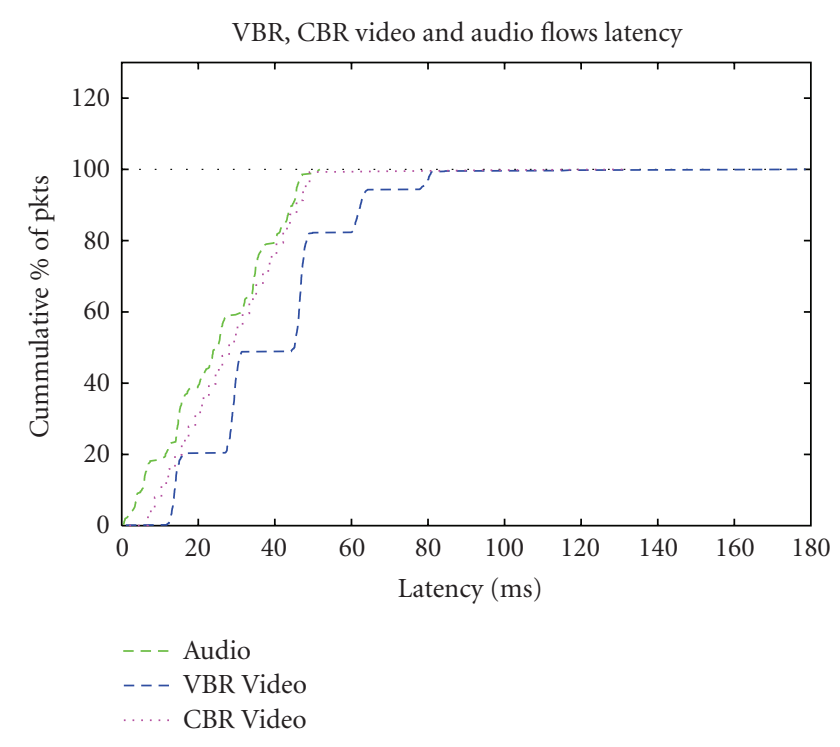

FIgURE 11: Latency distribution for AFDSA.

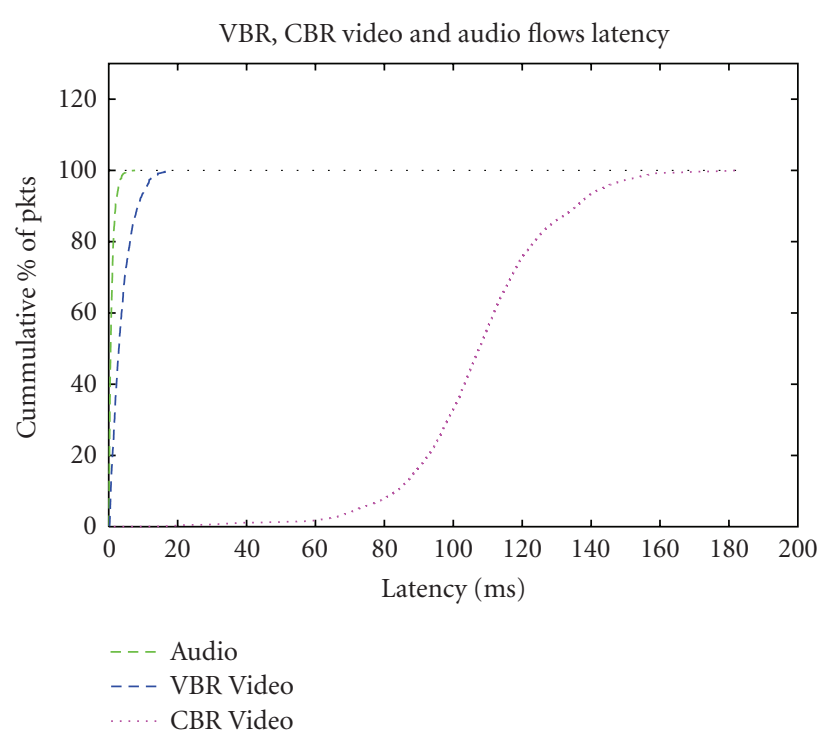

Figure 12: Latency distribution for EDCA.

simultaneously so the mean arrival time is 26 milliseconds. With 34 milliseconds interarrival time, the arrival times repeat with a period near $400 \mathrm{~ms}$. Therefore, packets can only get some specific latency between 0 and 50 milliseconds which causes a stair-shape latency distribution curve.

Figures 13 to 16 show the latency during the time. As depicted in these figures, the latency of AFDSA and FHCF methods are better than others. HCCA has problem in VBR video traffic and EDCA has problem with CBR video. Also the jitter of different flows in different methods is summarized in Table 5. Although EDCA has very low jitter, it suffers from very high-dropped packet number as illustrated in Table 6.

Table 3 demonstrates the characteristics of the selected traffics. The different VBR flows have been obtained with

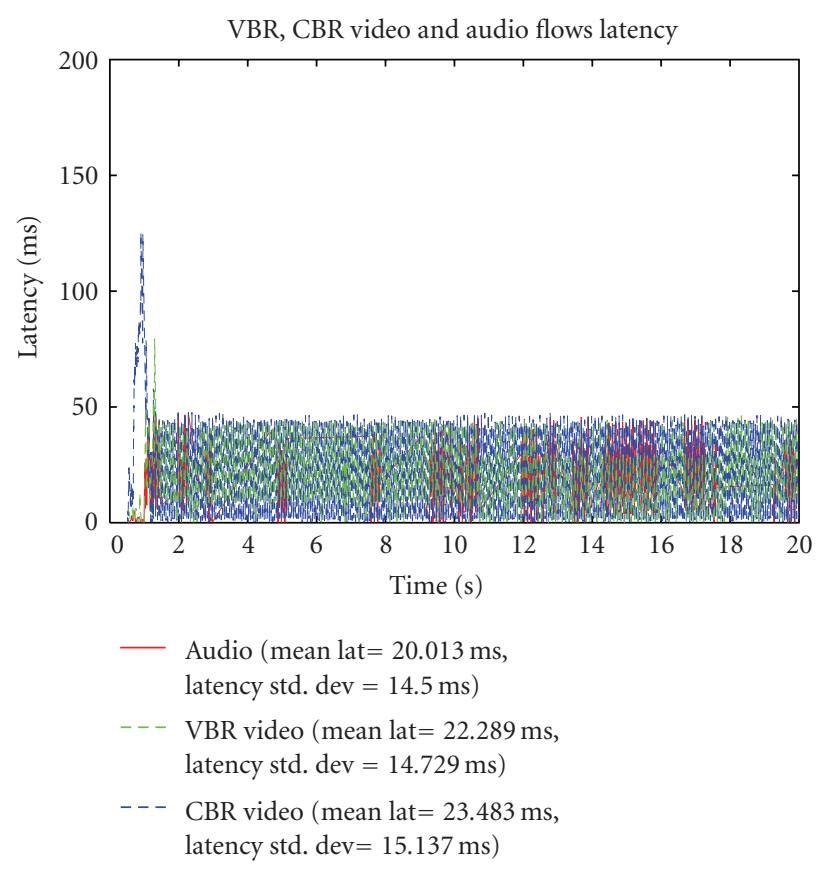

FIgURE 13: FHCF latency.

TABLE 6: Dropped-packet number for different methods.

\begin{tabular}{lcccc}
\hline & HCCA & FHCF & AFDSA & EDCA \\
\hline Audio & 0 & 0 & 0 & 0 \\
VBR Video & 0 & 0 & 0 & 0 \\
CBR Video & 108 & 91 & 101 & 6794 \\
\hline
\end{tabular}

VIC video-conferencing tool using the H.261 coding and QCIF format for typical "head and shoulder" video sequence. The PHY and MAC layer parameters used in the simulation are also summarized in Table 4 .

There are two methods to increase the channel load: increasing the node number and increasing the packet size. The latter is selected for increasing the channel load in this simulation. It is a time-consuming method because CBR video packets need more time to be transmitted. The packet size of CBR MPEG4 video has been increased from 600 to 1000 bytes to achieve $96 \%$ channel load.

Figure 17 shows fairness for VBR and CBR video traffic streams when the load increases up to $96 \%$. In order to compare the fairness of the different schemes for the same kind of traffic, Jain's fairness index has been employed [18]:

$$
J=\frac{\left(\sum_{i=1}^{n} d_{i}\right)^{2}}{n \sum_{i=1}^{n} d_{i}^{2}}
$$

where $d_{i}$ is the mean delay of the flow $i$ and $n$ is the number of flows. Figure 17 indicates that FHCF and AFDSA are fairer than HCCA.

\subsection{Scenario 2}

In scenario 2 (see Table 5), there are 7 nodes, six of which are sources and another is destination. Each QSTA has three 


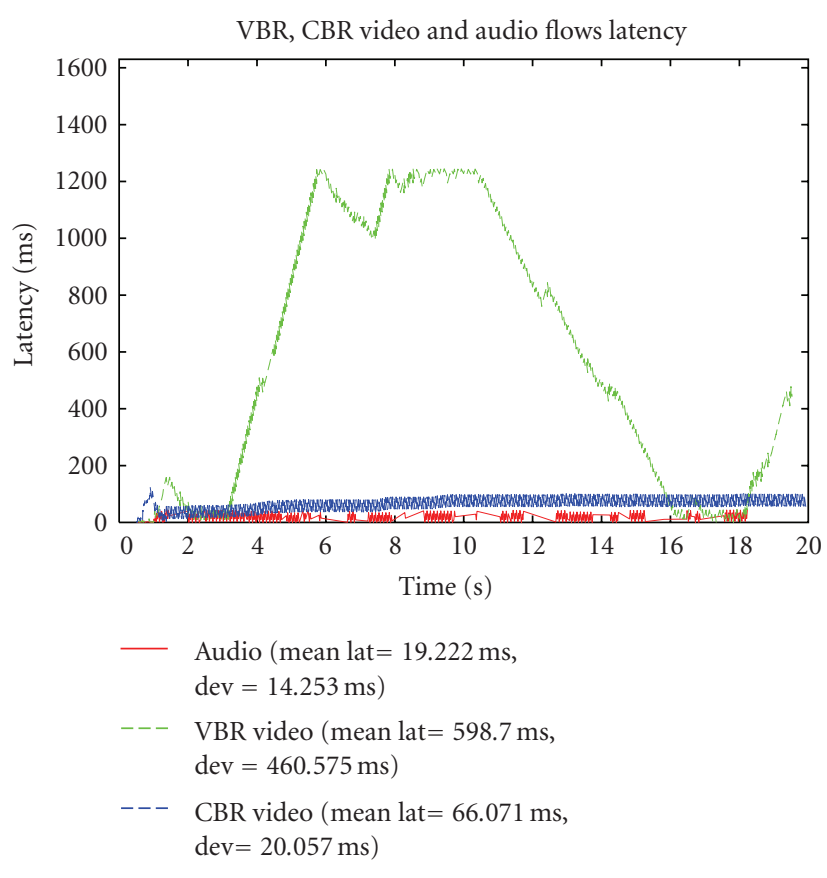

Figure 14: HCCA latency.

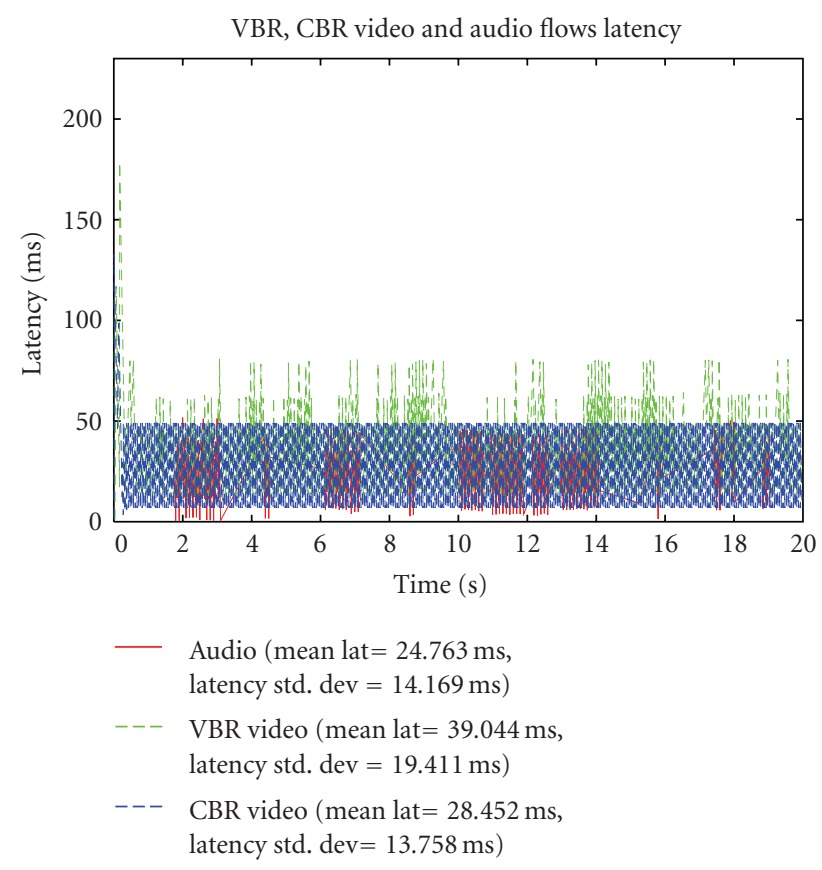

Figure 15: AFDSA latency.

different traffic flows (audio, VBR H.261 video, and CBR MPEG4 video flows) simultaneously through three different MAC layer priority classes. We increase the channel load by increasing the packet size of CBR MPEG4 traffic from 600 bytes $(2.4 \mathrm{Mbps}$ ) to 1000 bytes ( $4 \mathrm{Mbps}$ ) using a 100 bytes increment and keeping the same interarrival period of 2 milliseconds.

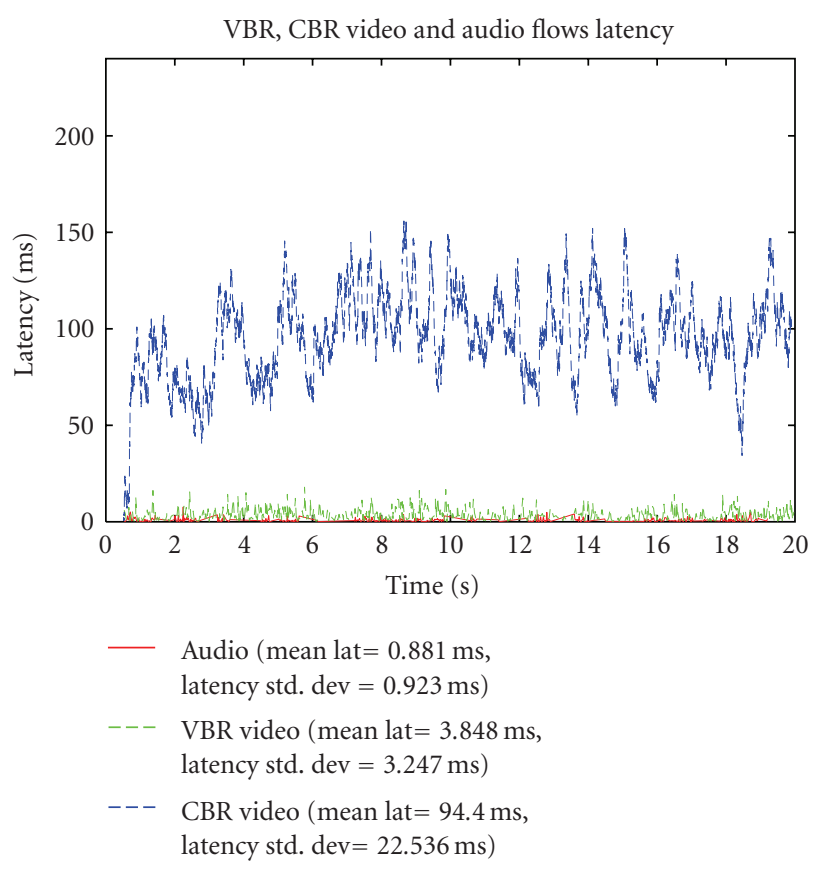

Figure 16: EDCA latency.

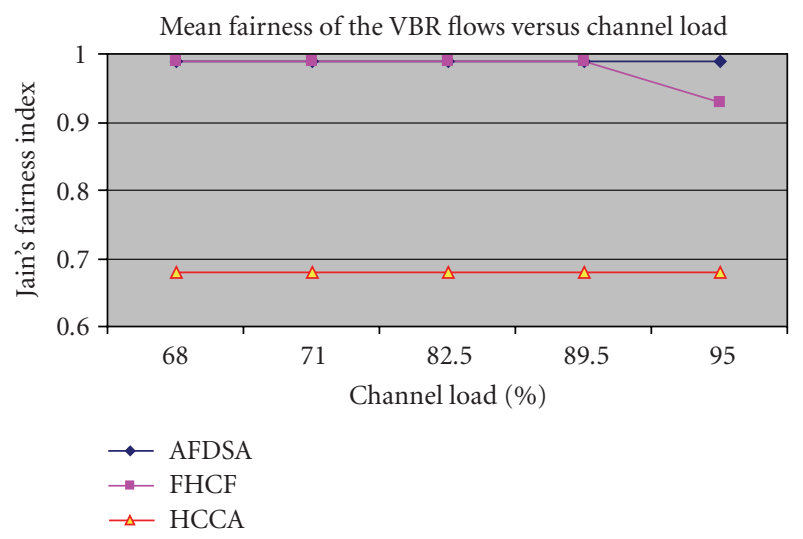

(a)

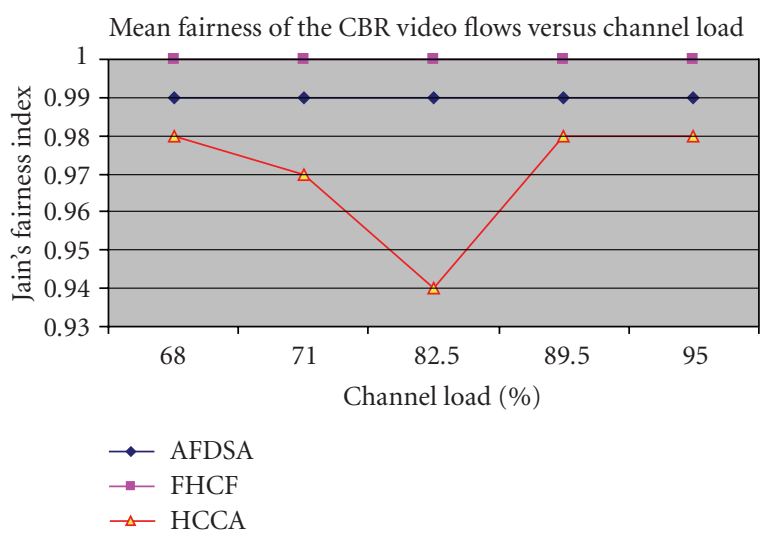

(b)

FIGURE 17: Mean fairness for VBR and CBR flows. 


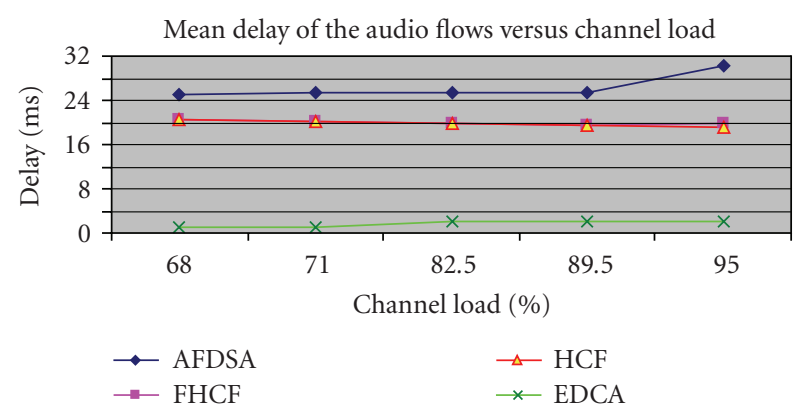

(a)

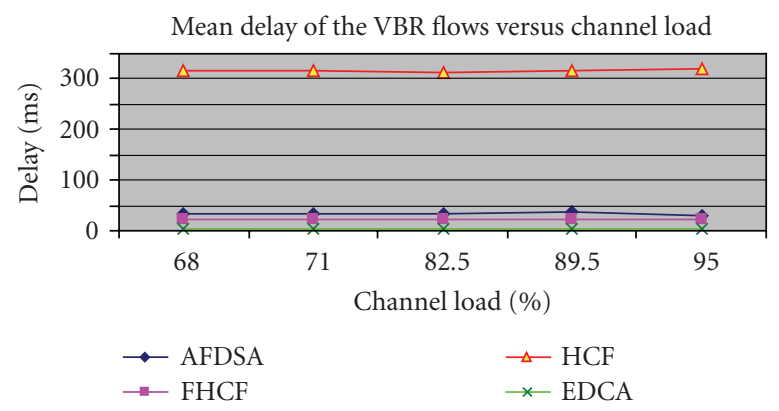

(b)

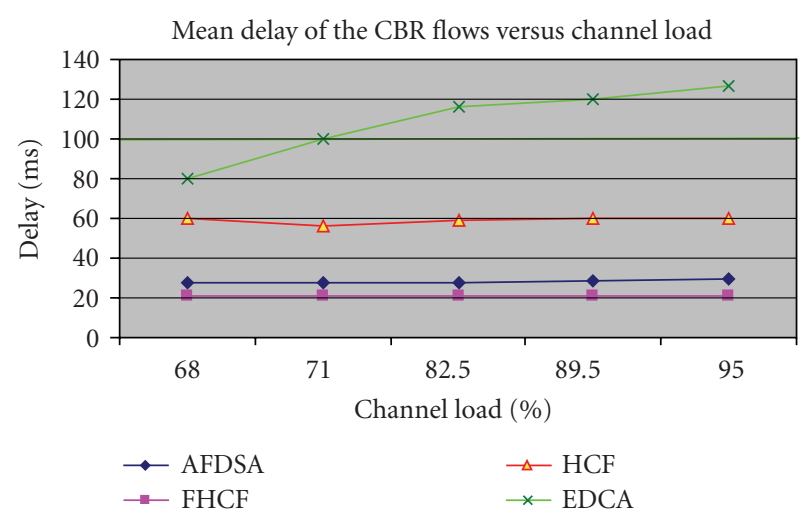

(c)

FIGURE 18: Mean latency for different flows versus channel load.

Figures 18 and 19 show the mean delay and fairness of several types of flows, obtained with the various schemes, for different loads of network, respectively.

\section{Audio and VBR H.261 video flows}

Figure 18 shows that the delay is almost constant for the FHCF and the AFDSA with increase in the load which indicates that delay does not strongly depend on the network load. In HCCA, VBR traffic has a high value of delay (300 milliseconds) that exceeds the limit for this kind of traffic.

In EDCA, mean latency is very low for audio and VBR video traffic streams because of the high priority that had been assigned for these streams. This increases the delay of CBR video traffic and it linearly increases with increase in traffic load. Figure 19 shows Jain index for all four methods. These methods are almost similar for audio traffic. It is

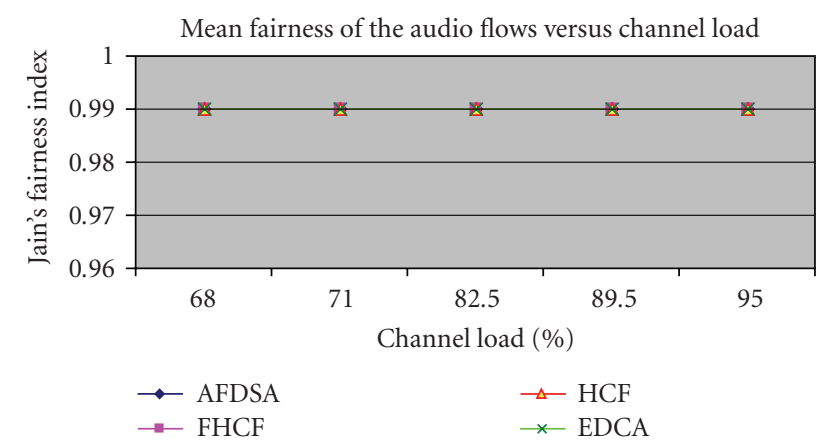

(a)

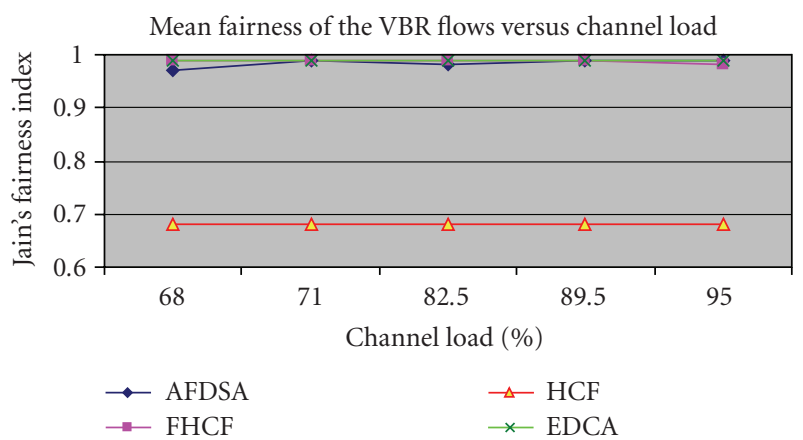

(b)

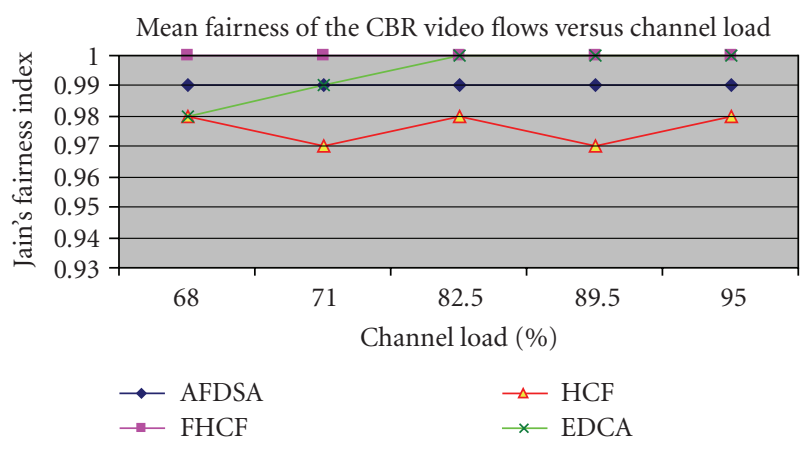

(c)

FIgURE 19: Mean fairness of different flows versus channel load.

apparent that AFDSA and FHCF are better than EDCA and HCCA for VBR video traffic.

\section{CBR MPEG4 video flows}

In our simulation, CBR streams are responsible for increasing the traffic load. As we can see in Figure 18, latency is almost constant for HCCA, FHCF, and AFDSA but increases with the load increment for EDCA such that for loads more than $79 \%$ it exceeds the limit for CBR traffic (100 ms). Figure 19 shows that Jain's index for all methods is high with minor differences for CBR video traffic.

\section{CONCLUSION}

A new distributed MAC scheduling algorithm (AFDSA) for upcoming 802.11e standard is proposed and evaluated. 
The mechanism introduces three additional fields to the RTS/CTS frame to guarantee QoS. The EDCA method of 802.11 e is used to access the channel for the first time. When time duration is reserved for a station, the rest of the stations only compete for accessing the channel in the unreserved periods. It is shown through extensive simulation that the AFDSA can guarantee QoS for both CBR and VBR video traffic. It does not need any point coordinator and each node can play an access point role if it is connected to the backbone.

\section{REFERENCES}

[1] IEEE 802.11 WG, "IEEE std. 802.11e, Part 11: Wireless MAC and physical layer specifications: MAC Quality of Service Enhancements," Reference number ISO/IEC 15802-3, November 2005.

[2] M. Malli, Q. Ni, T. Turletti, and C. Barakat, "Adaptive fair channel allocation for QoS enhancement in IEEE 802.11 wireless LANs," in Proceedings of IEEE International Conference on Communications (ICC '04), vol. 6, pp. 3470-3475, Paris, France, June 2004.

[3] J. Zhao, Z. Guo, Q. Zhang, and W. Zhu, "Distributed MAC adaptation for WLAN QoS diffrerentiation," in Proceedings of IEEE Global Telecommunications Conference (GLOBECOM '03), vol. 6, pp. 3442-3446, San Francisco, Calif, USA, December 2003.

[4] L. Romdhani, Q. Ni, and T. Turletti, "Adaptive EDCF: enhanced service differentiation for IEEE 802.11 wireless ad hoc networks," in Proceedings of IEEE Wireless Communications and Networking Conference (WCNC '03), vol. 2, pp. 1373-1378, New Orleans, La, USA, March 2003.

[5] G. W. Wong and R. W. Donaldson, "Improving the QoS performance of EDCF in IEEE 802.11e wireless LANs," in Proceedings of IEEE Pacific RIM Conference on Communications, Computers, and Signal Processing (PACRIM '03), vol. 1, pp. 392-396, Victoria, Canada, August 2003.

[6] W. Pattara-Atikom, S. Banerjee, and P. Krishnamurthy, "ADRAFT: an adaptive QoS mechanism to support absolute and relative throughput in 802.11 wireless LANs," in Proceedings of the 7th ACM Symposium on Modeling, Analysis and Simulation of Wireless and Mobile Systems (MSWiM '04), pp. 117-125, Venezia, Italy, October 2004.

[7] J. F. Lee, W. Liao, and M. C. Chen, "A MAC-layer differentiated service model in IEEE 802.11e WLANs," in Proceedings of IEEE Global Telecommunications Conference (GLOBECOM '05), vol. 6, pp. 3290-3294, St. Louis, Mo, USA, November-December 2005.

[8] Q. Deng and A. Cai, "A TXOP-based scheduling algorithm for video transmission in IEEE 802.11e networks," in Proceedings of the 6th International Conference on ITS Telecommunications (ITST '06), pp. 573-576, Chengdu, China, June 2006.

[9] C. Liu and C. Zhou, "Providing quality of service in IEEE 802.11 WLAN," in Proceedings of the 20th IEEE International Conference on Advanced Information Networking and Applications (AINA '06), vol. 1, pp. 817-822, Vienna, Austria, April 2006.

[10] E.-C. Park, D.-Y. Kim, C.-H. Choi, and J. So, "Improving quality of service and assuring fairness in WLAN access networks," IEEE Transactions on Mobile Computing, vol. 6, no. 4, pp. 337-350, 2007.

[11] J. Jackson Juliet Roy, V. Vaidehi, and S. Srikanth, "A QoS weight based multimedia uplink scheduler for IEEE 802.11e
WLAN," in Proceedings of the International Conference on Signal Processing Communications and Networking (ICSCN '07), pp. 446-451, Chennai, India, February 2007.

[12] P. Ansel, Q. Ni, and T. Turletti, "FHCF: a simple and efficient scheduling scheme for IEEE 802.11e wireless LAN," Mobile Networks and Applications, vol. 11, no. 3, pp. 391-403, 2006.

[13] A. Grilo, M. Macedo, and M. Nunes, "A scheduling algorithm for QoS support in IEEE802.11E networks," IEEE Wireless Communications, vol. 10, no. 3, pp. 36-43, 2003.

[14] G. Boggia, P. Camarda, L. A. Grieco, and S. Mascolo, "Feedback-based bandwidth allocation with call admission control for providing delay guarantees in IEEE 802.11e networks," Computer Communications, vol. 28, no. 3, pp. 325337, 2005.

[15] B. A. Venkatakrishnan and S. Selvakennedy, "An enhanced HCF for IEEE 802.11e wireless networks," in Proceedings of the 7th ACM Symposium on Modeling, Analysis and Simulation of Wireless and Mobile Systems (MSWiM '04), pp. 135-142, Venezia, Italy, October 2004.

[16] S. Montazeri, R. Berangi, and M. Fathy, "A new distributed scheduling algorithm to guarantee QoS parameters for 802.11e WLAN," in Proceedings of the International Conference on Information Networking (ICOIN '06), vol. 3961 of Lecture Notes in Computer Science, pp. 132-145, Sendai, Japan, January 2006.

[17] P. M. Soni and A. Chockalingam, "Performance analysis of UDP with energy efficient link layer on Markov fading channels," IEEE Transactions on Wireless Communications, vol. 1, no. 4, pp. 769-780, 2002.

[18] R. Jain, The Art of Computer Systems Performance Analysis, John Wiley \& Sons, New York, NY, USA, 1991. 